\title{
Probing Ensemble Polymorphism and Single Aggregate Structural Heterogeneity in Insulin
}

\section{Amyloid Self-Assembly}

Giuseppe De Luca ${ }^{a,+}$, Dirk Fennema Galparsoro ${ }^{a,+}$, Giuseppe Sancataldo ${ }^{a}$, Maurizio Leone $^{a}$, Vito Foderă ${ }^{b^{*}}$ and Valeria Vetri ${ }^{a, *}$

${ }^{\text {a }}$ Dipartimento di Fisica e Chimica, Università degli Studi di Palermo, Viale delle scienze Edificio 18, 90128 Palermo, Italy

${ }^{b}$ Department of Pharmacy, Faculty of Health and Medical Sciences, University of Copenhagen, Universitetsparken 2, 2100, Copenhagen, Denmark

KEYWORDS: Amyloid structure, heterogeneity, spherulites, Thioflavin T, , human insulin, Fluorescence lifetime imaging, Micro-FTIR, visible and subvisible particles 


\section{ABSTRACT}

Ensembles of protein aggregates are characterized by a nano- and micro-scale heterogeneity of the species. This diversity translates into a variety of effects that protein aggregates may have in biological systems, both in connection to neurodegenerative diseases and immunogenic risk of protein drug products. Moreover, this naturally occurring variety offers unique opportunities in the field of protein-based biomaterials. In the above-mentioned fields, the isolation and structural analysis of the different amyloid types within the same ensemble remain a priority, still representing a significant experimental challenge. Here we address such complexity in the case of insulin for its relevance as biopharmaceutical and its involvement in insulin-derived amyloidosis. By combining Fourier Transform Infrared Microscopy (micro-FTIR) and fluorescence lifetime imaging microscopy (FLIM) we show the occurrence, within the same ensemble of insulin protein aggregates, of a variable $\beta$-structure architecture and content not only dependent on the species analyzed (spherulites or fibrils), but also on the position within a single spherulite at submicron scale. We unambiguously reveal that the surface of the spherulites are characterized by $\beta$-structures with an enhanced H-bond coupling compared to the core. This information, inaccessible via bulk methods, allows us to relate the aggregate structure at molecular level to the overall morphology of the aggregates. Our findings robustly solve the problem of probing the ensemble and single particle heterogeneity of amyloid samples. Furthermore, they offer a unique, scalable and ready-to-use screening methodology for in-depth characterization of self-assembled structures, being this translatable to material sciences, drug quality control and clinical imaging of amyloid-affected tissues. 


\section{Introduction}

Over the last decades, understanding protein self-assembly has been a huge task in fields as material sciences, neuroscience and drug developments. Indeed, due to their biocompatibility and ease of functionalization, protein self-assembled structures have revealed their high potential for design and realization of materials for drug delivery ${ }^{1}$ and tissue engineering ${ }^{2-4}$ as well as electronics and water purification ${ }^{5-8}$. Notwithstanding these interesting features, protein aggregates may represent a bottleneck for efficient formulations of specific protein drugs, since their occurrence can critically compromise the dosing of the specific treatment, potentially increasing the immunogenic risk ${ }^{9}$. Finally yet importantly, a range of neurodegenerative disorders such as Alzheimer's and Parkinson's diseases are associated with aggregates of normally soluble proteins into elongated structures, named amyloid fibrils ${ }^{10-12}$.

Amyloid fibrils are characterized by cross $\beta$-structures stabilized by H-bonds. This highly repetitive structural arrangement is responsible for the physico-chemical properties and stability of the fibrils ${ }^{13}$. Interestingly, amyloid fibrils do not have a unique morphology, giving rise to the so-called amyloid polymorphism: ${ }^{14}$ although sharing common features, different fibril polymorphs may indeed present dissimilarities at the nano-, micro- or mesoscopic level. This is likely due to a variety of distinct but simultaneous intermediate steps occurring within the same aggregation reaction ${ }^{15}$. Differences in morphology often reflect different molecular arrangements and physicalchemical features ${ }^{16-22}$. Variations in 1) the number of protofilaments constituting the mature fibril, 2) the relative arrangement of the protofilaments and 3) the molecular structure of the protofilament are reported as the main sources of such variability ${ }^{23,24}$. While representing an opportunity in material science in terms of tunability of the structures for specific applications ${ }^{3,25}$, such a diversity in morphology poses a serious challenge when it comes to relate the subtle changes 
in the $3 \mathrm{D}$ arrangement to the specific biological (mis)function. This is true in protein drug development and immunogenity risk profile of protein particles, field in which a more thorough analysis of particle characteristics is now required by the authorities ${ }^{26}$ as well as in neurodegenerative diseases. In the latter case, this has indeed led to a massive effort to identify various amyloid morphotypes and connect them to different etiological subtypes of diseases ${ }^{23,27,28}$. Either one looks at them in vitro or in vivo, multiple fibril morphologies can simultaneously occur within the same protein ensemble, making the isolation and structural analysis of the different amyloid types experimentally challenging ${ }^{23,29}$.

To make more intricate this already complex picture, other amyloid-like species (named superstructures) may occur, exhibiting a significantly different $\beta$-sheet packing when compared to fibrils $\mathrm{s}^{30-33}$. Among others, amyloid-like spherical aggregates, named spherulites, ranging from few micrometers to millimeters in diameter can be formed both in vivo and in vitro ${ }^{34,35}$. This aggregate is characterized by a fascinating core-shell morphology and seems to be the result of a self-assembly pattern, which is common for proteins, synthetic polymers and minerals ${ }^{33,36,37}$. Independent of their originating element, spherulites show a peculiar pattern named "Maltese cross" when imaged via cross-polarized optical microscopy, which makes them readily detectable. Protein spherulites have been observed in a number of protein systems including insulin, lysozyme, amyloid $\beta$ peptide and $\beta$-lactoglobulin (BLG), they occur simultaneously with amyloid fibrils and are associated to Alzheimer's disease $\mathrm{e}^{34,38,39}$. Spherulites have a unique structure with a geometrical arrangement lying in between soft matter materials and crystals, in which protein flexibility coexists with a structural rigidity, conferred by nano-confined and ordered $\beta$-structures ${ }^{40}$. As for fibrils, protein spherulites can be functionalized showing potentials for drug release applications ${ }^{4}$. They are detected in insulin drug formulations ${ }^{26}$ and have tunable properties ${ }^{41}$. Regarding the last 
point, we have recently shown the possibility to form insulin spherulites with distinct morphologies, different molecular structures and physical properties by controlled selfassembly ${ }^{35}$. Interestingly, order-disorder transitions in the formation of protein spherulites may modulate the bio-crystallization of mineral structure ${ }^{40}$. When it comes to the analysis of protein spherulites-containing samples, the main challenge is the intrinsically heterogeneous nature of both the structure of a single spherulite (i.e. core-shell morphology) as well as the simultaneous occurrence of different types of spherulites within the same ensemble ${ }^{42,43}$.

From the above facts, it is clear that bulk analysis of amyloid formation (e.g. light scattering or Thioflavin $\mathrm{T}$ fluorescence) may mask both the ensemble heterogeneity (spherulites and fibrils) and the intrinsic structural heterogeneity at the single particle level (different types of spherulites + different types of fibrils). Therefore, methods of analysis of single aggregates are highly desirable. To this aim, a series of effective approaches mainly based on force microscopy ${ }^{44}$, microfluidics ${ }^{45}$, single molecule fluorescence microscopy ${ }^{46}$, have been developed over the recent years. While providing unprecedented hitherto information on the overall ensemble heterogeneity, in most of the cases, these approaches require specific sample preparations including deposition on specific surfaces ${ }^{47}$ or, in general, conditions away from the ones in which the aggregates have grown. This may potentially alter the physical properties of the structures, affecting the characterization of the sample. Importantly, these approaches mainly apply for the detection of amyloid fibrils ${ }^{14}$ and oligomeric species ${ }^{47}$, leaving unsorted the detection and characterization of other species significantly present in the ensemble. Finally, extracting information about the mesoscopic physical parameters as viscosity and order of the structure with high spatial resolution may require time-consuming processing of the data. In this context, advanced optical microscopy methods have already shown their potential as they give the possibility to analyze spatially 
heterogeneous samples, ${ }^{48,49}$ reveal intrinsic features of aggregates ${ }^{50,51}$ and separate multiple species occurring in protein aggregation ${ }^{52,53}$.

In the present work, we address the long-standing problem of disentangling the simultaneous occurrence of different amyloid species, from nano- to micro-scale, and readily identify in solution the presence of diverse morphotypes within the same ensemble without any sample manipulation. We specifically focus on human insulin (HI) aggregation, for its relevance in pharmaceutical industry and for its involvement in amyloid aggregate formation at the insulin injection sites during therapy (named insulin-derived amyloidosis) ${ }^{54}$. Insulin aggregation pathway is indeed considered among the most variegated in vitro aggregation reactions. Depending on the physico-chemical conditions of the formulation, insulin may self-assemble into different structures, from the nm to the $\mathrm{mm}$ range and with protein molecules spanning different conformational states ${ }^{35,39,55}$. These parameters may critically determine the immunogenic response induced by the insulin drug product $^{26}$. For this reason, tailored experimental approaches are urgently needed to properly retrieve information on the balance between different concurring aggregates, analyze their features and identify the immunogenic potency for each structure. This would in turn help the pipeline for the drug pre-formulation steps to enhance physical and chemical stability.

Coupling FTIR-microscopy with confocal fluorescence microscopy and fluorescence lifetime imaging, we map the heterogeneity of an insulin spherulite-containing sample, both at the level of spherulite-to-spherulite variation and structural heterogeneity of a single spherulite. Via FLIM analysis of Thioflavin T (ThT) fluorescence and by means of the phasor approach ${ }^{56}$, we also identify distinct molecular features of "below resolution" amyloid fibrils, giving a comprehensive and complete picture of the entire range of amyloids populations. 


\section{Results and discussion}

\section{Ensemble heterogeneity and aggregate-to-aggregate variability}

The analysis of a heterogenous ensemble of protein aggregates (i.e. fibrils + spherulites) as well as the intrinsic spatial heterogeneity in the structure at the level of single aggregate requires the application of methods that allow spatial localisation of spectroscopic measurements. To this aim, we exploit the potential of correlating structural information from micro-FTIR to the exquisite sensitivity of fluorescence microscopy and fluorescence lifetime imaging. We use ThT as a fluorescence probe for its ability to increase its quantum yield when it is bound to amyloid aggregates. This feature, with some care/restrictions can be used to qualitatively evaluate the amount of amyloid material in the aggregated samples ${ }^{57,58}$.
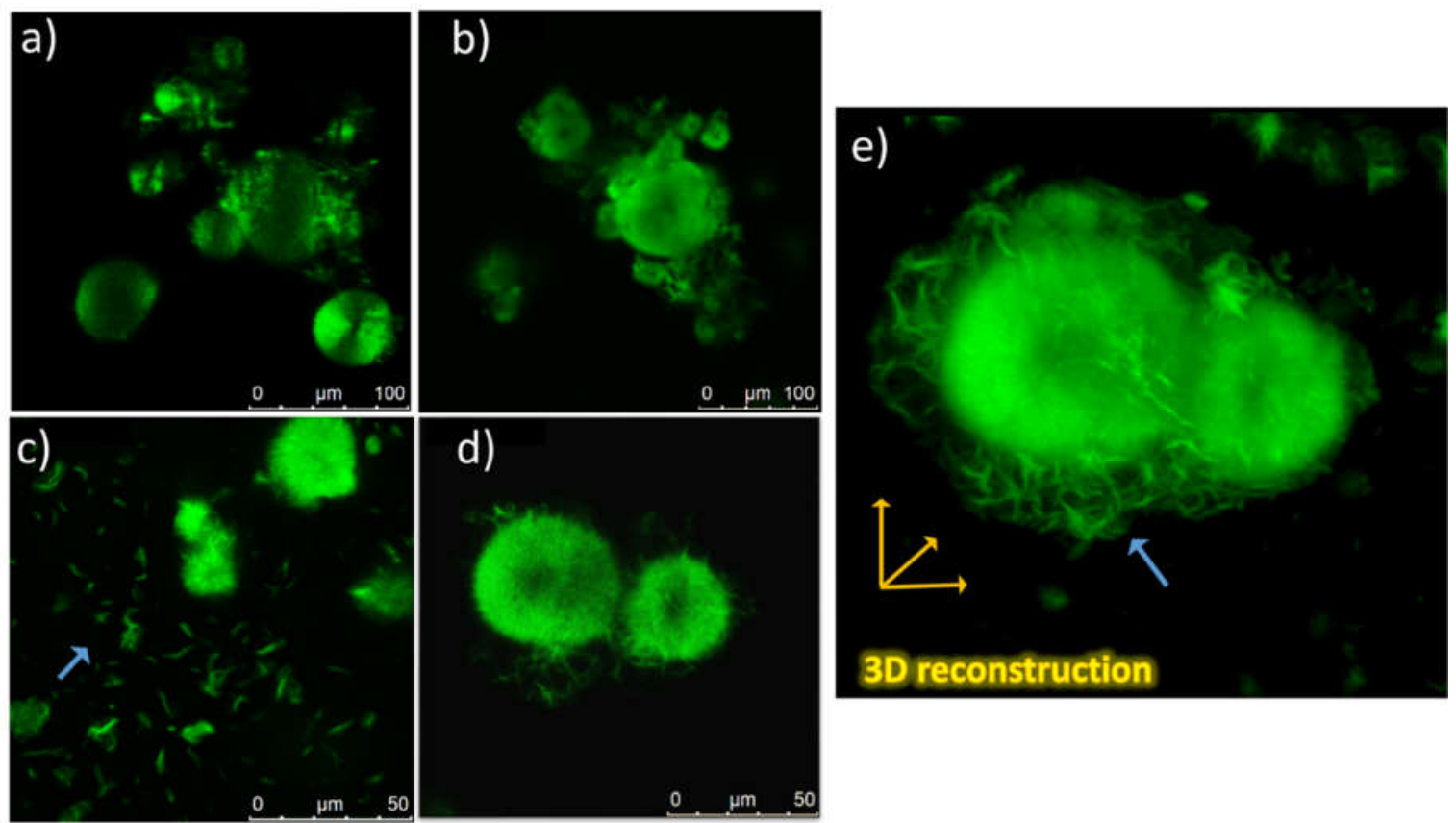

Figure 1. Representative fluorescence images of $\mathrm{HI}$ aggregates formed by incubating a $5 \mathrm{mg} / \mathrm{ml}$ $\mathrm{HI}$ in $\mathrm{HCl}$ solution ( $\mathrm{pH} \mathrm{1.85)} \mathrm{and} 0.25 \mathrm{M} \mathrm{NaCl}$ at $60^{\circ} \mathrm{C}$ for 24 hours and stained with ThT. a-d) $1024 \times 1024$ pixels representative images showing the large sample heterogeneity: both amyloid 
spherulites and fibrils (cyan arrow in panel c) are present. e) 3D reconstruction of spherulites structure. A lower fluorescence intensity is detected in the internal region of the spherulites compared to the edge; fibrils (cyan arrow) are also detected and are characterised by slightly higher intensity.

In figure 1a-d we report 1024 x 1024 pixels representative images of a typical HI sample after incubation at $60^{\circ} \mathrm{C}$ and $\mathrm{pH} 1.85$ for 24 hours. These experimental conditions are widely used to study in vitro insulin aggregation ${ }^{35}$. The micrographs perfectly summarize the complexity we face: a mixture of spherical aggregates (spherulites) of different sizes and with different capability of being stained by ThT (i.e. difference in the ThT intensity among different spherulites and different regions of the spherulites can be qualitatively observed (Figure 1a-c) together with isolated fibrils (Figure 1c,e cyan arrow). The diameter range of the observed spherulites is between few and hundreds of micrometers and their appearance resembles the classical core-shell morphology, with fibril-like structures elongated from a central core. Interstingly, the 3D reconstruction of a group of two spherulites surrounded by fibrils (Figure 1e, see Supplementary Information, SI, for the video) better highlights that ThT fluorescence intensity along the spherulite structure is not constant. It presents a minimum (nearly vanishing fluorescence) at the center/core of the structure, suggesting a pronounced structural heterogeneity even within a single spherulite (See Figure S1 in SI). Moreover, amyloid-like fibrils are again detected along the entire volume scanned (Figure 1e, cyan arrow). ThT fluorescence intensity may depend on both affinity and accessibility of binding sites. While affinity is mostly depending on the binding site structure specificity (i.e. presence of $\beta$-sheet), the accessibility is dependent on both the specific geometrical arrangement of the aggregate structure and the solvent conditions. 
Due to the characteristic size of spherulites compared to the ones of fibrils, one can qualitatively conclude that the formation of spherulites is not a minor pathway in insulin aggregation. More quantitatively, in the case of bovine insulin and in conditions similar to the ones used in the present study, approximately $80 \%$ of the total protein molecules is converted into spherulites $^{38}$, emphasizing once more the importance to analyze this aggregation pathway to have a realistic understanding of the whole aggregation process. Notwithstanding this, spherulites are rarely described. This may be due to the fact that the kinetics of spherulite formation are indistinguashable from the one for fibrils ${ }^{35,38,55,59}$. Moreover, due to their micrometric size, they readily sediment and their presence may be hindered if the sample is not correcly handled. As an example, sample preparations with dilution and drying procedures, as for the transmission electron microscopy or atomic force microscopy, tend to irreversibly destroy their structures. For human insulin in analogous conditions, the presence of spherulites has been previously highlighted via approaches with no or minor sample treatment ${ }^{60}$. These aggregates are ideal samples for optical microscopy techniques and can be imaged directly after formation; their 3D spatial organisation can be readily investigated in aqueous solutions without further sample treatment, either by cross polarized microscopy or ThT fluorescence microscopy $y^{35,38}$.

We analyze different spherulites using micro-FTIR. In Figure 2 we report representative optical images of the sample (Figure 2, a-e) where the heterogeneity in size and morphology is evident. Several large structures indeed occur: some of them are single large objects, others are formed by clustering of multiple structures, this being in line with confocal microscopy measurements. For each image, we select $50 \mu \mathrm{m}$ x $50 \mu \mathrm{m}$ regions of interest (ROIs) within the different spherulites (colored squares in Figure 2a-e). This technique allows to distinguish 
secondary structural differences in selected areas of these large protein aggregates. Due to spatial resolution of the image and low contrast, fibrillar structures are not detectable by micro-FTIR.

FTIR spectra of the Amide I' region $\left(1575 \mathrm{~cm}^{-1}-1710 \mathrm{~cm}^{-1}\right)$ acquired in the highlighted ROIs and after normalization are reported in Figure $2 \mathrm{f}$ and $2 \mathrm{~g}$, respectively (following the color code). These measurements allow one to obtain detailed information on the conformation of the protein backbone in a specific region. Moreover, a detailed analysis of the band can provide 1) the reciprocal orientation of $\beta$-strands forming the sheet in amyloids (parallel $v s$ antiparallel), 2) the strength of inter-strand hydrogen bonds or the number of strands composing the sheet and 3) the twist and the hydration state of the polypeptide chains of strands ${ }^{61}$. In the case of protein aggregates, a peak centered at about $1620 \mathrm{~cm}^{-1}$ is considered a fingerprint of amyloid structures ${ }^{61-}$ 64.
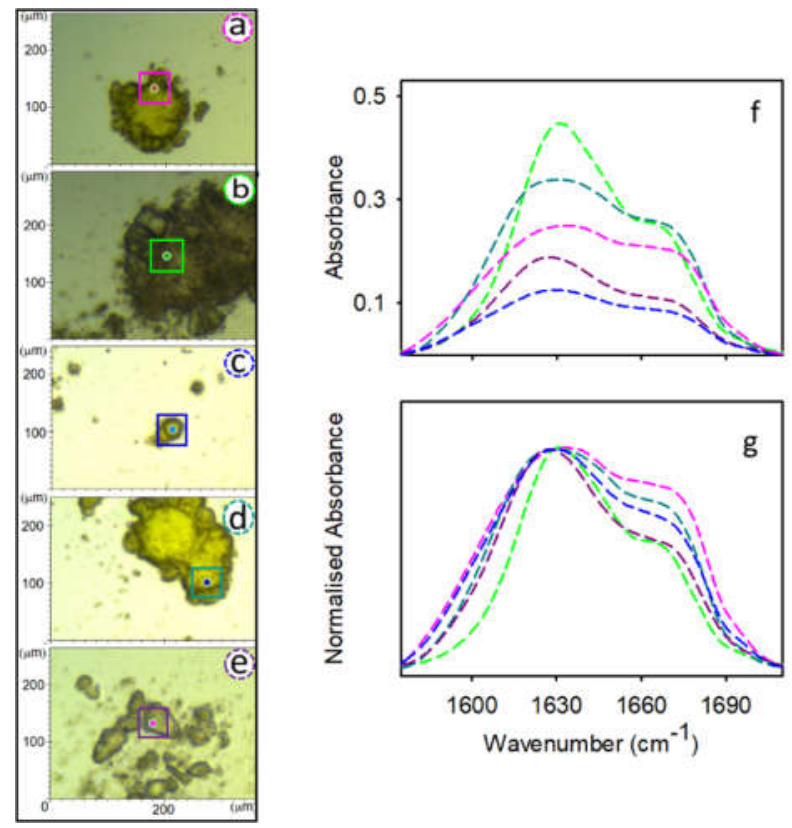

Figure 2 Ensemble heterogeneity of an HI spherulites sample. a-e): Representative optical images of HI spherulites. The colored $50 \mu \mathrm{m}$ x $50 \mu \mathrm{m}$ squares represent the ROIs where FTIR spectra were acquired. Light path is $17 \mu \mathrm{m}$. f) FTIR spectra in the Amide I' region $\left(1575 \mathrm{~cm}^{-1}-1710 \mathrm{~cm}^{-}\right.$ 
${ }^{1}$ ) acquired in each ROI of each image following the same color code. g) FTIR spectra normalised by their maximum value.

As shown in Figure $2 \mathrm{f}$ and $2 \mathrm{~g}$, a quite pronounced heterogeneity, visible via optical microscopy, is also reflected at the level of the secondary structure. Even if the FTIR spectra from different spherulites share common features, clear differences in both the shape and intensity of the Amide I' band emerge. For each spherulite, the spectrum is mainly characterised by two large peaks centered at $1620 \mathrm{~cm}^{-1}$ and one at $1670 \mathrm{~cm}^{-1}$, respectively. In line with previous reports ${ }^{49-52}$, the $1620 \mathrm{~cm}^{-1}$ peak is ascribable to the aggregated $\beta$-sheets characteristic of the amyloid structure, while the other broad peak can arise from the presence of native and aggregate structures including $\alpha$-helices, turns and loops $\left(1660 \mathrm{~cm}^{-1}\right)$ and antiparallel aggregated $\beta$-sheets $\left(1670-1680 \mathrm{~cm}^{-1}\right)^{49-52}$. As the spherulites are contained in a sample holder with fixed $17 \mu \mathrm{m}$ pathlength, differences in the intensity of the Amide I' peak according to Lambert Beer's law, stem for the different density of the protein molecules within the spherulite in the ROIs. Moreover, the relative ratio between the two peaks is not the same for the areas analyzed, indicating that the balance between native-like and aggregate structural components is variable among spherulites within the same sample. Interestingly, the bulk spectrum of the aggregated sample in the SI (Figure S2) presents a more pronunced peak at $1620 \mathrm{~cm}^{-1}$ compared to the one observed in figure 2 as both spherulites and amyloid fibrils contribute to this peak, being the latter excluded when using the micro-FTIR setup. The FTIR spectrum of native insulin in the same conditions is also reported, confirming an initial dominant presence of $\alpha$-helices and only a minimal amount of $\beta$-sheets ${ }^{65}$. The comparison between the two acquisition methods highlights the non ergodic behaviour of the presented sample. 
Normalised spectra in Figure $2 \mathrm{~g}$ allow the qualitative evaluation of the differences between spheulites structures at secondary structure level. As can be seen, changes are not only detected in the ratio between the two main peaks $\left(1620 \mathrm{~cm}^{-1}\right.$ and $\left.1670 \mathrm{~cm}^{-1}\right)$, but also in the width of the two main peaks, depending on the spherulite under investigation. This indicates that each subcomponent contributes to the spectrum in different extent and each structure presents different levels of heterogeneity. Interestingly, the most pronounced spectral differences are detected in the lower wavenumbers region (below $1630 \mathrm{~cm}^{-1}$ ). The spectral component centered at about 1620 $\mathrm{cm}^{-1}$ accounts for the intermolecular $\beta$-sheets in amyloid structure and the peak position may vary depending on the strenght of the H-bonds and on the lenght of the $\beta$-chains. Indeed a shift toward lower wavenumbers occurs when hydrogen bond strength in $\beta$-aggregate structures increases, or when the number of $\beta$-sheets grows ${ }^{61,63}$. This translates into the fact that a narrow peak would stem for an overall structure characterized by a negligible variability in terms of intermolecular bonding, while a large peak indicates the presence of multiple structures with different molecular packing and robustness. It is worth noting that different levels of $\mathrm{H}_{2} \mathrm{O} / \mathrm{D}_{2} \mathrm{O}$ exchange may also affect the individual spectral component in Amide I/I' band depending on the level of solvent accesibility ${ }^{63}$. However, this would only be a minor effect since the overall shape of the peak is similar and present the same features both in small $(<25 \mu \mathrm{m}$ diameter $)$ and large $(>150 \mu \mathrm{m}$ diameter $)$ spherulites. Altogether, data in Figure 2 point out the pronounced ensemble heterogeneity with the co-existence of not only spherulites with different sizes, but also with significant differences in 1) the relative content of $\beta$ structures compared to native-like structures, 2) the $\beta$-sheets strenght and 3) their intermolecular packing.

Intrinsic structural heterogeneity at single aggregate level revealed by micro-FTIR 
Due to the core-shell nature of spherulites, we cannot rule out that the spherulite-tospherulite structural differences in Figure 2 can arise by an intrinsic spatial heterogeneity within the same spherulite. In Figure $3 \mathrm{a}$ we report a 460 × $350 \mu \mathrm{m}$ optical image of a single large spherulite. To investigate the structural heterogeneity of the spherulite, we acquired FTIR spectra in the $50 \mu \mathrm{m} \times 50 \mu \mathrm{m}$ ROIs indicated by the squares in the figure, from the inner to the external part of the spherulite and report them in Figure 3b. We also report the spectra normalized by the native like component at $1668 \mathrm{~cm}^{-1}$ in Figure 3c. As can be seen, the intensity and shape of the Amide I' present a gradual variation depending on the position on the spherulite structure. The absorption peak intensity has its maximum at positions closer to the spherulite center and decreases when moving toward the peripheral regions (Figure $3 \mathrm{~b}$ ). This suggests that the protein density within the aggregate has its highest value at the center of the spherulite, while decreases when approaching the edge of the structure. Spectra normalized by the native like component at 1668 $\mathrm{cm}^{-1}$ (Figure 3c) also reveal that the amount of $\beta$-aggregate structures increases when moving from the center of the spherulite to the edges, areas in which the $\beta$-aggregates content becomes dominant. Within the same sample, we observe this trend independently of the size of the spherulites (see SI figure S3 and S4).
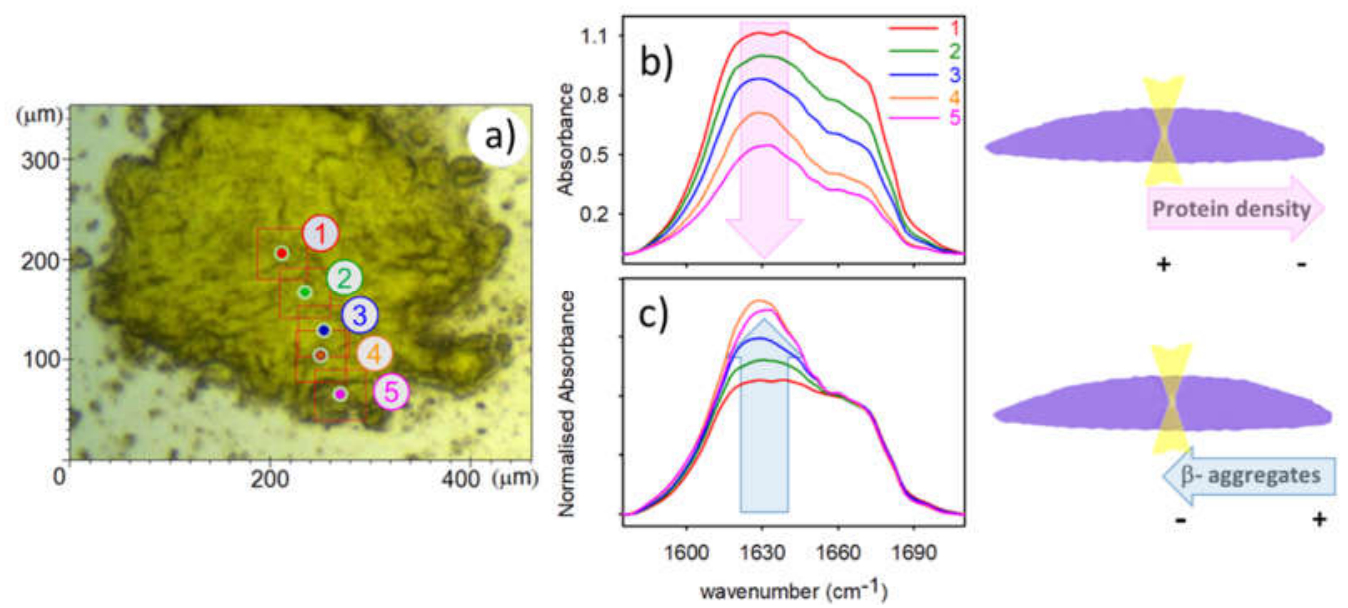
Figure 3. Structural differences within a spherulite: a) $460 \mu \mathrm{m} \times 350 \mu \mathrm{m}$ optical image of a single large spherulite. The red $50 \mu \mathrm{m}$ x $50 \mu \mathrm{m}$ squares represent the ROIs where FTIR spectra were acquired from the center 1) to the external part of the spherulites 5). Light path is $17 \mu \mathrm{m}$. b) FTIR spectra in the Amide I' region $\left(1575 \mathrm{~cm}^{-1}-1710 \mathrm{~cm}^{-1}\right)$ acquired in the ROIs of each image, c) FTIR spectra normalized at the value at $1668 \mathrm{~cm}^{-1}$.

Figure $4 \mathrm{a}$ shows the deconvolution of the spectrum nr. 5 in Figure 3 using 4 components, namely $1603 \mathrm{~cm}^{-1}, 1620 \mathrm{~cm}^{-1}, 1640 \mathrm{~cm}^{-1}$ and $1670 \mathrm{~cm}^{-1}$ with a spectral width of $20 \mathrm{~cm}^{-1}$. This heuristic choice is aimed at comparing relative amounts of different structures (and not at identifying all possible secondary structure components in the sample) $)^{52}$. The $1620 \mathrm{~cm}^{-1}$ and the $1603 \mathrm{~cm}^{-1}$ bands are assigned to amyloid aggregate structures. The component at $1603 \mathrm{~cm}^{-1}$ is useful for the fit description. Indeed, its growth describes the presence of larger amounts of intermolecular $\beta$ structures components with stronger H-bonds or longer $\beta$-chain. Finally, the peak at $1640 \mathrm{~cm}^{-1}$ is assigned to native-like components, while the peak at $1670 \mathrm{~cm}^{-1}$ is related to the presence of nonspecific turns and loops.

In Figure $4 \mathrm{~b}$ the fractional areas of the aggregated component of the spectra (obtained as the sum of the fractional areas of the $1603 \mathrm{~cm}^{-1}$ and $1620 \mathrm{~cm}^{-1}$ ) and of the native-like component (obtained as the sum of the fractional area of the $1640 \mathrm{~cm}^{-1}$ and $1670 \mathrm{~cm}^{-1}$ ) are shown. The details of the percentage fractions with respect to the total Amide I' area for each component and for each spectrum are also reported in Figure 4c. 

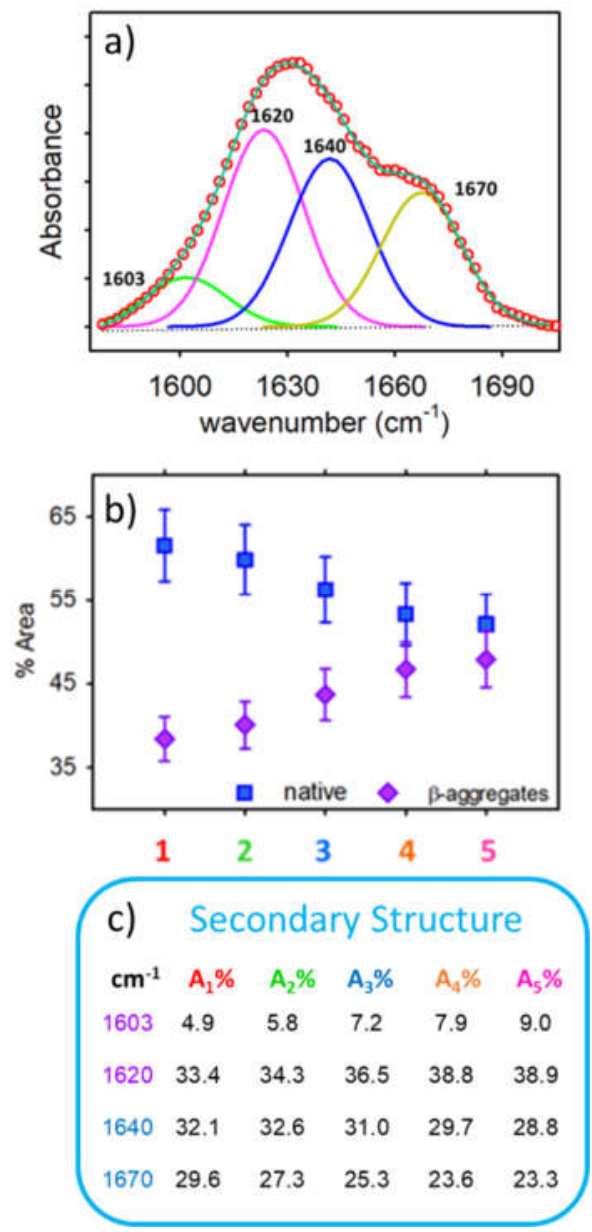

Figure 4. a) Representative spectral deconvolution in Gaussian components of FTIR spectrum of the ROI nr. 5 in figure 3. Selected spectral components are centered at $1603 \mathrm{~cm}^{-1}, 1620 \mathrm{~cm}^{-1}, 1640$ $\mathrm{cm}^{-1}$ and $1670 \mathrm{~cm}^{-1} .20 \mathrm{~cm}^{-1}$ Gaussian width is kept constant. b) Fractional areas of the spectral components related to the aggregates and native-like protein (see text for the definition) as a function of the position in the spherulite: from internal (1) to the external (5) regions. c) Fractions of the total area of the Amide I' band for each of the component used in the deconvolution. Moving away from the spherulite center, a correlation exists between the reduction of native-like structure and the appearance of the aggregate structure. The ratio between $1603 \mathrm{~cm}^{-1}$ and $1620 \mathrm{~cm}^{-1}$ components also increases when approaching the spherulite edges.

A correlation between the reduction of the native-like fraction and the growth of $\beta$-aggregates is evident. Moreover, the ratio between the $1603 \mathrm{~cm}^{-1}$ and $1620 \mathrm{~cm}^{-1}$ area increases while moving away from the spherulite center (see Figure 4c). This result can be interpreted as a progressive shift or broadening of the amyloid peak toward lower wavenumbers confirming that the H-bonds coupling grows in the external regions of the spherulites. 
The above reported results clearly show that the structural heterogeneity within a single spherulite can be closely related to a well-defined spatial heterogeneity of the aggregate morphology. Our experiments indicate that the dense core of a spherulite is rich in native-like structure. Moving away from the central part, an increasing amount of $\beta$-structures is detected. Moreover, the $\beta$-structures gradually change their nature while moving away from the core, having a more pronounced $\mathrm{H}$-bonds strength close to the edges of the spherulites. These results are in line with a previous micro-Raman based work highlighting the significant presence of amorphous material at the center of insulin spherulites responsible for the absence of birefringence when spherulites are imaged via cross-polarized microscopy ${ }^{42}$. Taken together, our findings show that coupling imaging analysis and spectroscopy data provides details on the spatial distribution of different aggregated structures. Future advances in technologies may also allow circular dichroism microscopy ${ }^{66}$ on amyloid systems, which may constitute a good opportunity for the analysis of nm- size species with an increased spatial resolution.

Mapping the intermolecular architecture of amyloid aggregates via fluorescence lifetime microscopy

Micro-FTIR results are in line with the observation reported in Figure 1 showing that ThT fluorescence intensity is not uniform within a single spherulite. ThT intensity is indeed dimmer at the center and brighter in the external part. As mentioned above, the difference in ThT intensity at different spherulite locations can be due to: 1) different affinity of the dye to the structure, 2) different number of available binding sites for ThT, 3) different accessibility of these binding sites $^{67}$ and 4) differences in the quantum yield (QY) of ThT modulated by different molecular environments in its surroundings ${ }^{58,68}$. Combining the fluorescence microscopy data and the micro- 
FTIR analysis, we can safely conclude that the highest ThT fluorescence signals are detected away from the core of the spherulite, where the $\beta$-aggregate components are dominant. Moreover, in the central areas containing a reduced content of $\beta$-aggregate, we detect a low ThT fluorescence signal. Not surprisingly, this confirms that the intensity of ThT fluorescence well correlates with the presence of $\beta$-aggregate. However, the fluorescence of ThT may also contain further useful information on the aggregate structure. ThT is a molecular rotor and its fluorescence signal growth is likely determined by the steric hindrance of the internal rotation of the ThT aromatic rings relative to each other, which blocks the transition to the non-fluorescent state ${ }^{69}$. This feature makes ThT quantum yield also dependent on the solvent viscosity and on the rigidity of its microenvironment ${ }^{68,69}$. In addition, ThT binds perpendicularly to the long axis of the fibril and the dimethylamino group of the molecule interacts with the fibril surface. This means that different structural properties of the $\beta$ aggregate structure (e.g. chain length or H-bond strength) may modify the binding mode of ThT to amyloid structures ${ }^{70-72}$. Specifically, charge, presence of specific residues (e.g. aromatics) or spacing between the $\beta$-strands may alter the binding to $\mathrm{ThT}^{73}$.

Based on the above facts, the detected ThT fluorescence properties are the result of differences in affinities between the dye and binding sites and on the ThT interaction with the environment. Thus a detailed analysis on the ThT signal may provide information on the different binding modes or the physical properties of the environment experienced by the dye. However, the evaluation of the ThT intensity alone would not allow such an in depth analysis. ThT fluorescence lifetime can give separate indications of different binding modes and, in turn, on the differences in the amyloid structures. Indeed, different architectures in $A \beta$ amyloid fibrils were shown to lead to different ThT fluorescence lifetimes ${ }^{74}$ and, recently, Kuimova and coworkers reported the formation and stabilization of micron scale structures accompained by an increase in 
the ThT fluorescence lifetime, which they attributed to an increase in the effective viscosity of the environment in the proximity of ThT molecules ${ }^{50}$. Importantly, fluorescence lifetime measurements present the great advantage of not being dependent on the chromophore concentration (i.e. not depend on the number of molecules occupying the binding sites).

We perform ThT fluorescence lifetime imaging analysis of single insulin spherulites. Specifically, we report FLIM analysis of measurements acquired at two different Z-quotes of the sample, i.e. close to the equatorial line (Figure $5 \mathrm{a}-\mathrm{d}$ ) and at the bottom (Figure $5 \mathrm{e}-\mathrm{g}$ ) of a spherulite. At the bottom of the sample smaller species, like fibrils, that precipitate in solution can also be found. FLIM measurements reported in the following are analysed using the phasor $\operatorname{approach}^{56,75}$. This method provides a global view of fluorescence molecules decays at each pixel in the images not imposing any specific model as required by fitting procedures ${ }^{49,56,75,76}$. When using phasor analysis each pixel of the intensity images is mapped to a point in the phasor plot corresponding to the measured fluorescence lifetime. Single exponential lifetimes lie on the socalled "universal circle"; long lifetimes are located near the origin ( 0 on the $\mathrm{x}$ axis), while short lifetimes are shifted on the circumference toward the bottom right intersection with the $\mathrm{x}$ axis (1 on the $\mathrm{x}$ axis) ${ }^{49,56}$.

In Figure 5a and 5e we present 256x256 pixels ThT fluorecence intensity maps of a spherulite (diameter of $\sim 50 \mu \mathrm{m}$ at the center of the field of view) at the equatorial plane and at the bottom of the structure, respectively. Importantly, other species, namely other spherulites and fibrils, are also detectable. The phasor plots corresponding to these measurements are shown in Figure $5 \mathrm{~b}$ and $5 \mathrm{f}$ together with magnifications of relevant areas (Figure 5c and 5g). Phasors lie inside the universal circle indicating that ThT lifetimes in these conditions are characterised by non-single exponential decay ${ }^{49,50,56}$. The presented analysis immediately reveals a large fluorescence lifetime distribution 
appearing as a cloud of points extended along a straight line in the phasor plot (see the orange dashed line in Figure $5 \mathrm{c}$ and $5 \mathrm{~g}$ ). In figure $5 \mathrm{~d}$ and $5 \mathrm{~h}$ pixels with specific lifetime selected in the phasor plot using colored circles are mapped back in the image using the same colors. We here qualitatively describe the large lifetime distibution in the phasor plot selecting 3 main components with the colored circles. As observed in Figure $5 \mathrm{~d}$ at the equatorial plane of the spherulite, the distribution with longer lifetimes (pink) is detected at the edge of the spherulites, while signal characterised by intermediate lifetime (green) is found in the central part of the spherulites .The component with shorter lifetimes (yellow) seems to be related to well distinct structures with fibrillike morphology being not part of the spherulites .
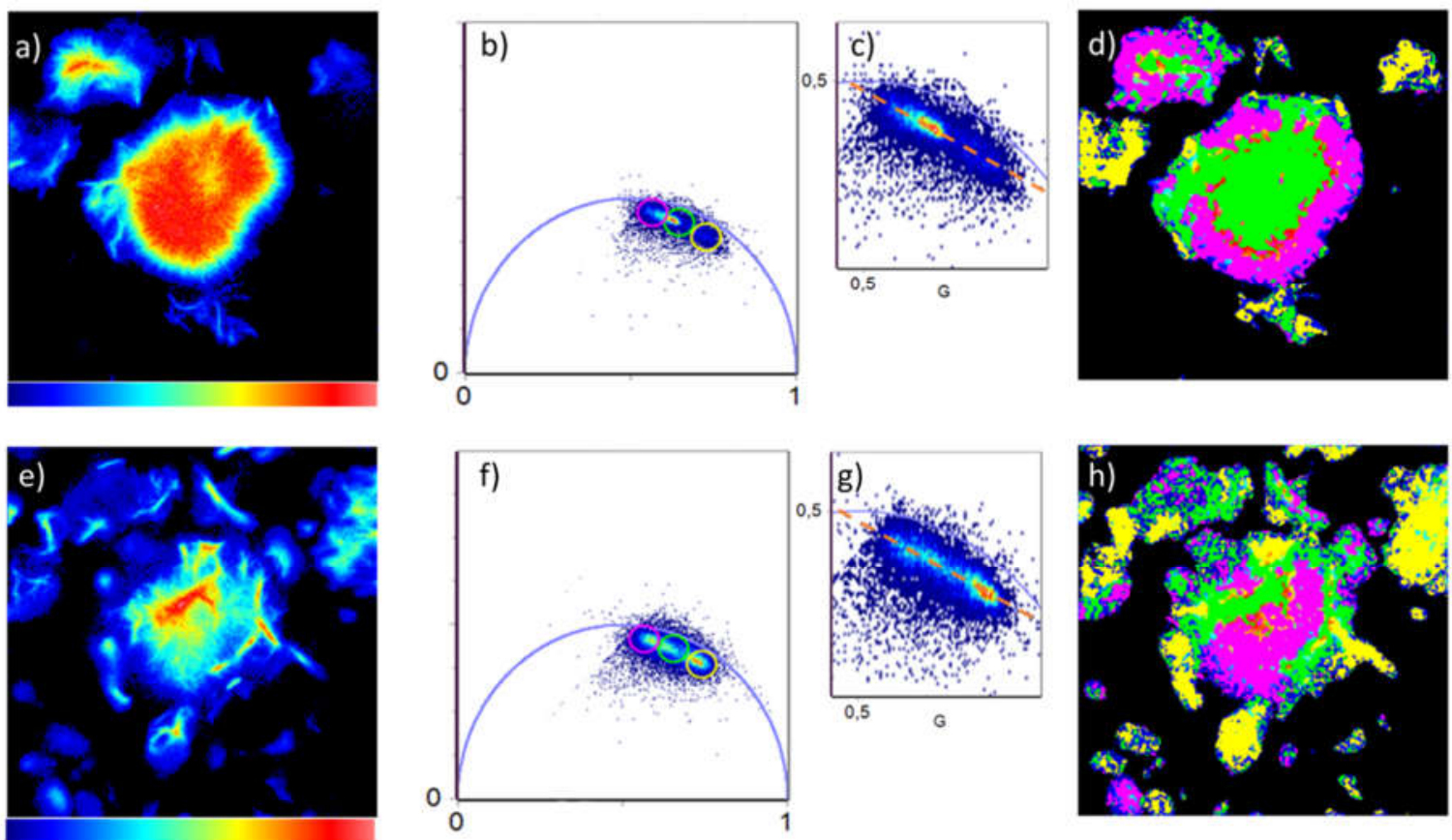
Figure 5. Phasor analysis of 256x256 pixels FLIM measurements on ThT signal at the equatorial plane a-d) and at the bottom e-h) of a spherulite stained with ThT. The sample is obtained by incubating $\mathrm{HI}$ at $5 \mathrm{mg} / \mathrm{ml}$ in $\mathrm{HCl}$ solution, $\mathrm{pH} 1.85$ and $0.25 \mathrm{M} \mathrm{NaCl}$ at $60^{\circ} \mathrm{C}$ for 24 hours. a) and e) fluorescence intensity images, from low (blue) to high (red) intensity, image size is $96.3 \mu \mathrm{m} \mathrm{x}$ $96.3 \mu \mathrm{m}$; b) and f) phasor plots obtained from measurements in a) and e), respectively. c) and g) magnifications of the region highlighting that distribution maximum shifts along a straight-line in the phasor plot (orange line is a guide to the eye). $d$ ) and h) Phasor color map: each pixel is colored according to the color of the corresponding circle in the phasor plot. The choice of the size and the position of the circles is arbitrary and it is used to highlight average properties of the lifetime distributions, which are located on the same line.

If one moves to the bottom of the spherulite, as highlighted in Figure $5 \mathrm{~g}$ ), we note that the lifetime distributions lie on the same line as in Figure $5 \mathrm{c}$ ), being the shorter lifetime component largerly populated. Using the same positions of coloured circles, yellow pixels are again related to fibril-like structures well distinct from the spherulite (Figure 5h). Green and pink pixels are located on the spherulite (Figure 5i), with a large part of the image covered by the pink distribution. This is not in contrast with the data in Figure 5a-d since this 2D section of the spherulite relates to an area that is close to the edge/surface of the structure. We then expect this area to behave as the external part of the spherulites, so that detecting areas with longer lifetimes seems to be a fingerprint of the surface properties. Importantly, the shorter lifetime distributions (yellow) in proximity of the spherulites edge (but not part of the structure), is located where fibrils are detected (see also SI, Figure S5). As a reference we also report in SM in Figure S6 the phasor plot of ThT self-fluorescence in conditions where it is not bound to amyloid structures. A unique lifetime distribution with relatively low value of lifetime is observed and is well separated from the distributions detected in the case of the ThT-spherulites system.

Data and interpretation presented in Figure 5 are fully supported by the quantitative analysis of a z-stack acquired on a single spherulite. In Figure 6 we report quantitative FLIM 
analysis of measurements acquired along z-axis for a single spherulite (see schematics in Figure 6a). In Figure $6 \mathrm{~b}$ the FLIM analysis of a section at the equatorial plane of the spherulite $(\mathrm{z}=14 \mu \mathrm{m}$, see also Figure S1 in SI for the optical section) is based on the decomposition of the phasor plot data using two principal lifetime components.

Indeed, the lifetime distribution cloud lies within the universal circle and on the same straight line as the one higlighted in the phasor plot in figure $5 \mathrm{c}$ and $5 \mathrm{~h}$. The data trend, then, suggests a double exponential decay and the two mono-exponential components can be identified via the intersection of the straight line with the universal circle ${ }^{50,56}$. This is based on the fact that phasors follow the vector algebra. Single lifetime species components are characterized by monoexponential decays and the related phasors lie on the universal circle. Phasor of complex species are the result of a linear combination of the single lifetime species components. A double exponential decay would lie within the universal circle and on the line connecting two monoexponential decays phasors. The distance between each point of the cloud in the phasor plot and the single exponential phasors on the universal circle $\left(F_{1}\right.$ and $F_{2}$ in Figure $\left.6 b\right)$ represents the fraction of each component ${ }^{53}$. In our case and according to the used calibration, it is possible to draw a straight line connecting the measured phasors to two points on the universal circles representing single exponential decays with $\tau_{1}=0.6 \mathrm{~ns}$ and $\tau_{2}=2.5 \mathrm{~ns}$ (green and red cursors in Figure $6 \mathrm{~b}) . \tau_{1}$ and $\tau_{2}$ are identified as principal components. The analysis of ThT fluorescence decays in terms of two components was previously performed in different systems ${ }^{50,77}$, this being the simplest model to describe the ThT fluorescence decay. The fractions $F_{1}$ and $F_{2}$ obtained for the measurement at the equatorial plane $(\mathrm{z}=14 \mu \mathrm{m})$ are mapped in false color in figure $6 \mathrm{c})$. The scale goes from blue (pure fast component at $\tau_{1}=0.6 \mathrm{~ns}$ ) to red (pure slow component at $\tau_{2}=2.5$ ns). In particular, data are reported in terms of fraction of the $\tau_{1}$ component. The fastest decay is 
dominant in small species not embedded into spherulite and resembling fibril-like material $\left(F_{1}=0.53\right)$, while the core shows a faster decay $\left(F_{1}=0.43\right)$ when compared to the external surface $\left(F_{1}=0.3\right)$. Similar maps are obtained for measurements at different quotes from the equatorial plane down to the peripheral part of the aggregates (Figure 6d) together with the corresponding phasor plots (Figure 6e). Approaching the bottom of the spherulites ( $\mathrm{z}=1 \mu \mathrm{m}$ and $\mathrm{z}=3 \mu \mathrm{m}$ ) the distribution characterizing the surface (i.e. with longer lifetimes) becomes dominant. The area corresponding to the edges of the spherulites appears colored in yellow-red in Figure $6 \mathrm{~d}$ ), this being in line with the idea that the entire external part of the spherulite has a marked difference compared to the internal part. Moreover, we note that at $\mathrm{z}=1 \mu \mathrm{m}$ the lifetime distribution attributed to fibril-like structures is well separated from the ones measured in the spherulites, as indicated by the arrow in the phasor plot; the corresponding pixels appear colored in cyan in Figure 6d highlighting regions where ThT is characterized by shorter lifetimes. 


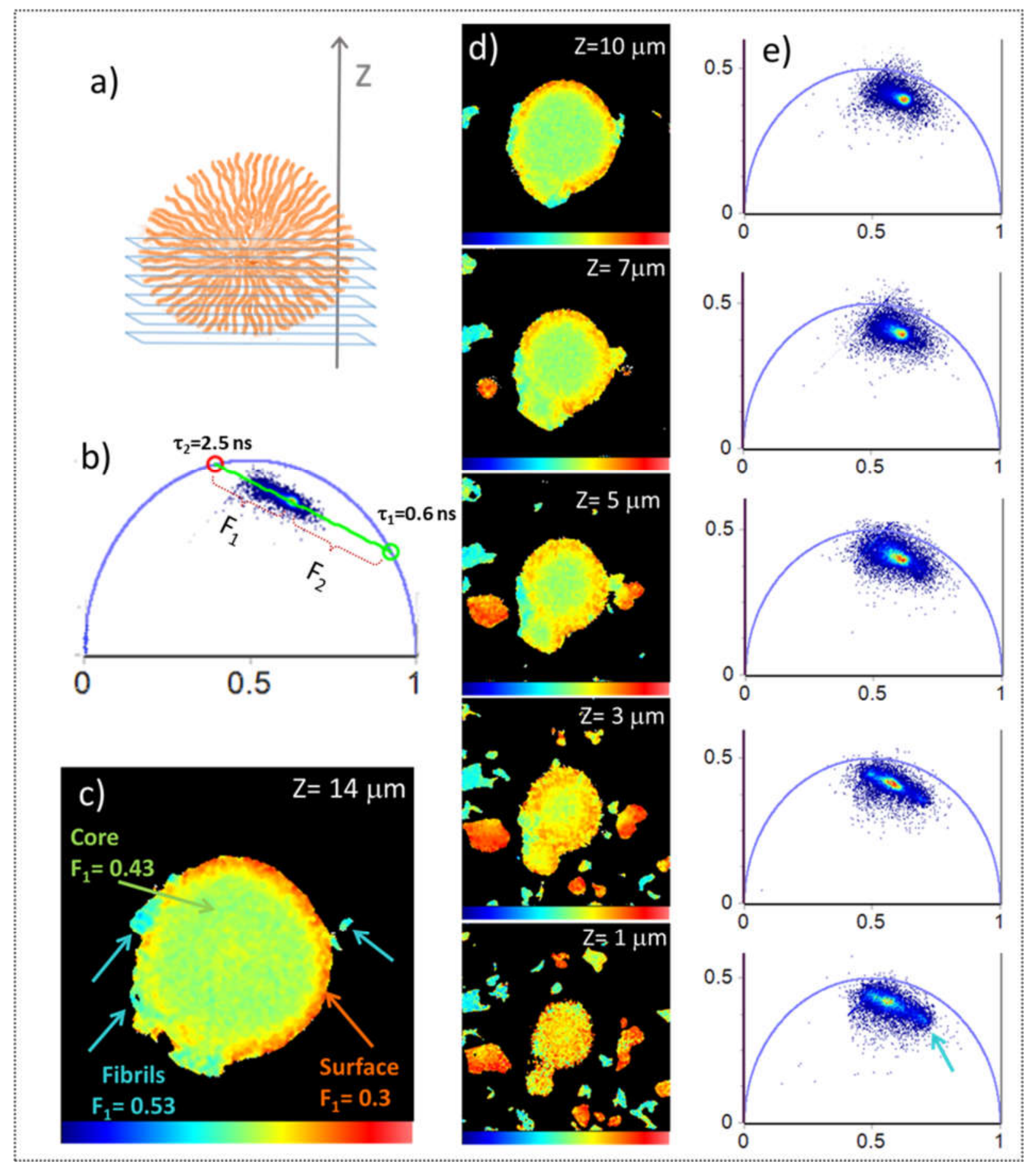

Figure. 6) Phasor analysis of 256x256 pixel FLIM measurements on ThT signal for a Z-stack from the bottom to the equatorial plane of a single spherulite. a) Schematics of image acquisition along the spherulite. b) Phasor plot from the analysis of the FLIM image at the equatorial plane ( $\mathrm{z}=14$ $\mu \mathrm{m})$. c) Lifetime fraction map for the FLIM image at the equatorial plane. Lifetime principal 
components are $\tau_{1}=0.6 \mathrm{~ns}$ (blue) and $\tau_{2}=2.5 \mathrm{~ns}$ (red). $\mathrm{F}_{1}$ is the fractional contribution of faster component. Image size is $96.3 \mu \mathrm{m}$ x $96.3 \mu \mathrm{m}$. d) lifetime fraction maps and phasor plots from the same spherulite at different z-quotes. e) Qualitative analysis of these measurements with same color code as Figure 6 is reported in Figure S7 in the SI.

ThT lifetime changes may be due to the superimposition of two main effects: the viscosity of the enviroment ${ }^{68,69}$ and the specificity of the ThT binding to the amyloid structures. While the first effect can be determined by the solvent and the overall density of proteins in the aggregate, the second one is likely to be dependent on the the detail of the $\beta$-sheet architecture around the ThT binding site ${ }^{69}$. In our specific case, the observed changes in the lifetimes may reflect variations in the aggregate structure as highlighted by the micro-FTIR measurements. Higher content of intramolecular $\beta$-structures on the edge of the spherulites (Figure 3) can indeed induce a modified topology of the ThT environment. This is reported for amyloid systems in which the formation and the stabilization of micron-scale structures lead to a shift of the lifetime distribution along a straight line in the phasor plot and it is attributed to a modified viscosity of the ThT environment ${ }^{50}$. However, the specificity of ThT towards intermolecular $\beta$-sheet structures stabilized by H-bonds with different strengths should also be considered. Our micro-FTIR data clearly suggest the occurrence at the surface of the spherulites of intermolecular $\beta$-structures with either stronger $\mathrm{H}$ bonds or longer $\beta$-chain compared to the ones in the internal part of the structure (Figure $4 b-c$ ). This means that different ThT binding modes/affinities may occur in the different areas of the spherulites, affecting the observed ThT lifetimes. More specifically, stronger H-bonds and/or longer $\beta$-chains would probably add more constraints and less flexibility to the ThT binding site with the result of inhibiting more efficiently the non-radiative ThT rotation and increasing the fluorophore's lifetime ${ }^{68}$. This picture is supported by Sidhu and co-workers, which employ the 
analysis of ThT fluorescence decays in terms of two components (both in the ns range) to discriminate among different fibril polymorphs ${ }^{78}$. Shorter lifetime components were attributed to ThT binding sites characterized by larger flexibility, while longer lifetime components to tighter binding sites. This means that observed differences in the ThT lifetimes between the aggregates (fibrils and spherulite) and within single spherulites (Figure 6) are related to a difference not only in the packing of $\beta$-sheet structure but also in the configuration of the single $\beta$-sheet structure. It remains to be clarified if these effects can only be attributed to modified inter-proteins interactions along the structures or they are mainly mediated by different level of hydration ${ }^{79,80}$.

\section{Conclusions}

Effective analysis of an ensemble of protein aggregates is strongly limited by the prononunced heterogeneity in species, which is a result of a multiplicity of interconnected aggregation pathways. Together with a large variety of high hierarchial structures, like fibrils and spherulites, a not negligible microscopic polymorphism of the species occurs. While potentially being a resource if one looks at amyloids in the context of materials ${ }^{3,25}$, the structural heterogeneity at different levels may translate into a complex biological heterogeneity when amyloids are investigated in relation to immunogenic risk of protein drug products ${ }^{9}$ or neurodegenerative diseases $^{12}$. All this calls for approaches capable of disentangling such complexity.

We prove that coupling micro-FTIR and time resolved fluorescence microscopy can be efficiently used as a tool for studying the intrinsic structural heterogeneity of the microscale aggregates at two different levels: 1) structural diversity and aggregate to aggregate variability (fibrils $v s$ spherulites) and 2) structural variability within the same aggregate. In the specific case 
of spherulites, our results reveal a pronounced spatial heterogeneity in the fluorescence lifetime distribution within a single spherulite. This arises from the heterogeneity of the structure as verified by micro-FTIR, which leads to differences in the amount of bound ThT molecules and on their quantum yield. Distributions of longer ThT fluorescence lifetime are observed in areas where FTIR $\beta$-structure signal is more intense and where more pronounced strength in H-bonds/long $\beta$-chains are found. Our results unambigously show that the core-shell morphology of the spherulites is related to a microstructure heterogeneity and that the surface has distinct properties compared to the internal part of the aggregate. Moreover, a clear distinction between free fibrils in the sample and the spherulites can be readily detected using ThT fluorescence and FLIM.

Besides sheding new light on the molecular architecture of insulin spherulites and their coexistence with fibrils, we demonstrate that our approach can be used as a simple, reproducible and artifact-free method of analysis of heterogeneous amyloid samples in solution without the need of invasive sample preparation and time-consuming data processing. Importantly, looking also at the lifetime rather than only the intensity of ThT fluorescence provides unreported information on the correlation between the fluorescence properties of this dye and the detailed molecular architecture of the stained amyloid aggregates ${ }^{21}$. The proposed approach can be applied in vitro for the detection of protein sub visible particles with size in the micrometer range this, being particularly important in pharmaceutical product development ${ }^{81}$. Moreoever, the possibility to easily translate this method to cell and tissue imaging may also pave the way to decipher the relation between aggregate structure and immunogeneicity potency and the identification of the features determining the risk profile of protein particles.

\section{Methods}

\section{Chemicals and Sample preparation}


Human Insulin (HI, 91077C-purity $\geq 90 \%$ ) and Thioflavin $\mathrm{T}$ (T3516-dye content $\geq 65 \%$ ) were obtained from Sigma-Aldrich and used without further purification. HI was dissolved in solution of $\mathrm{HCl}(\mathrm{pH} 1,85)$ (diluted from 37\% $\mathrm{HCl} 30721$-Sigma-Aldrich) and 0,25M NaCl (diluted from powder S7653- Sigma-Aldrich- purity $\geq 99.5$ ) at a protein concentration of $5 \mathrm{mg} / \mathrm{ml}$ and then the sample was filtered through $0.22 \mathrm{~mm}$ filters (MS 16534; Sartorius). Protein concentration was determined by UV absorbance at $276 \mathrm{~nm}$ using an extinction coefficient of 1.0 for $1.0 \mathrm{mg} / \mathrm{ml}$. Aggregation was thermally induced at $60{ }^{\circ} \mathrm{C}$ over a period of 24 hours in $1 \mathrm{ml}$ Eppendorf $(1.5 \mathrm{ml}$, polypropylene, 20170-038, VWR). 5 samples were formed for each experiment, repeating the process 3 times.

\section{Fourier Trasmission Infra Red (FTIR) spectroscopy and micro-FTIR.}

Aggregates were pelleted by centrifugation and re-suspended in $\mathrm{D}_{2} \mathrm{O}$ (Sigma-Aldrich 151882 , purity $\geq 99 \%$, Sigma Aldrich) for 3 times. After this, samples were left to equilibrate for 12 hours before measurements. Confocal microscopy measurements revealed that the treatment did not change the overall appearance of the sample and spherulites and fibrils were still present in the samples with no detectable changes.

Fourier transform infrared (FTIR) measurements in bulk were carried out with a Bruker Vertex 70 spectrometer equipped with a DTGS (doped triglycine sulfate) detector, in a sample compartment under continuum purging in $\mathrm{N}_{2}$ dry atmosphere. Aliquots of native $\mathrm{HI}$ and aggregate samples were exchanged in $\mathrm{D}_{2} \mathrm{O}$ and placed between two $\mathrm{CaF}_{2}$ windows separated by a $25 \mu \mathrm{m}$ Teflon spacer. Each final spectrum is an average of 128 scans in the $400-7000 \mathrm{~cm}^{-1}$ range with a spectral resolution of $2 \mathrm{~cm}^{-1}$. 
Micro-FTIR measurements were performed at room temperature using a LUMOS Fourier transform infrared microscope (Bruker), equipped with a photoconductive MCT detector with liquid nitrogen cooling. Visual image collection was performed via a fast digital CCD camera integrated in the instrument. Aliquots of aggregated samples diluted 1:50 in $\mathrm{D}_{2} \mathrm{O}$ were placed between two $\mathrm{CaF}_{2}$ windows separated by a $17 \mu \mathrm{m}$ Teflon spacer, this ensuring common path length for absorption measurements. FTIR spectra were acquired in transmission mode, between 4000 and $700 \mathrm{~cm}^{-1}$. Each final spectrum is the average of 128 scans. The variable microscope aperture was adjusted to squared region of interest with $25 \mu \mathrm{m} \times 25 \mu \mathrm{m}$ or $50 \mu \mathrm{m}$ x $50 \mu \mathrm{m}$ size.

We performed fittings of the Amide I' bands in terms of 4 Gaussian components after suitable baseline subtraction. The following expression was used:

$$
I(v)=\sum_{i=1}^{n} A_{i} \exp \left[-\left\{\left(v-v_{i}\right)^{2}\right\} / 2 \sigma_{i}^{2}\right]
$$

where $v_{i}, \sigma_{i}$ and $A_{i} \sigma_{i} \sqrt{2 \pi}$ are the peak frequency, width, and the area of the $\mathrm{i}^{\text {th }}$ component. The peak frequency of the selected four components was kept fixed at $1603 \mathrm{~cm}^{-1}, 1620 \mathrm{~cm}^{-1}, 1640 \mathrm{~cm}^{-}$ ${ }^{1}$ and $1670 \mathrm{~cm}^{-1}$ spectral width is fixed at $20 \mathrm{~cm}^{-1}$. We defined "fractional area" $\mathrm{A}(\%)=A_{i} \sigma_{i} \sqrt{2 \pi}$ $/ \sum_{i=1}^{n} A_{i} \sigma_{i} \sqrt{2 \pi}$. Each component is assigned to a specific backbone conformation; the fractional area indicates the relative amount of protein secondary structure in each spectrum. This standard type of analysis assumes identical extinction coefficients for the different structural element.

Two-photon excitation and Confocal Microscopy and Fluorescence lifetime imaging 
Spherulite samples were diluted 1:100 and stained with $26 \mu \mathrm{M}$ ThT. Five hundred microliters of stained samples were placed on microscope chambered slides and imaged at $1024 \times 1024$ pixels resolution using a Leica TCS SP5 confocal laser scanning microscope, using a $63 \times$ or $40 \times$ objective (Leica Microsystems, Germany). Importantly, we use protein/dye molar ratio so that possible effect of dye self-quenching previously observed for ThT can be ruled out ${ }^{82}$. Moreover, this choice is also based on our previous work on insulin aggregation, in which we proved that quencing effects are not significant at ThT concentration $\mathrm{ThT} \leq 40 \mu \mathrm{M}$ and in a wide range of protein concentrations $(1-20 \mathrm{mg} / \mathrm{ml})^{83}$.The two-photon excitation (Spectra-Physics Mai-Tai Ti:Sa ultra-fast laser) for ThT was set at $885 \mathrm{~nm}$ emission was detected in the range 450-500 nm, confocal imaging was performed using $\lambda_{\mathrm{ex}}=470 \mathrm{~nm}$ (Leica "white light laser) and fluorescence was detected in the range $\lambda_{\mathrm{em}}=485-585 \mathrm{~nm}$.

Fluorescence lifetime imaging measurements were acquired in the time domain by means of a picoHarp 300 standalone TCSPC module (Picoquant). $256 \times 256$ images were acquired at a scanning frequency of $400 \mathrm{~Hz}$, Leica "white light" laser was used in order to excite ThT $\left(\lambda_{\text {ex }}=470\right.$ $\left.\mathrm{nm}, \lambda_{\mathrm{em}}=485-585 \mathrm{~nm}\right)$.

\section{FLIM analysis}

FLIM data were analysed by the phasor approach ${ }^{49,56,75}$ by the SimFCS software developed at the Laboratory of Fluorescence Dynamics, University of California at Irvine (www.lfd.uci.edu). FLIM calibration of the system was performed by measuring the known lifetime of the fluorescein that is a single exponential of $4.0 \mathrm{~ns}^{84}$.

Phasor analysis is a Fourier domain technique, which allows the transformation of the fluorescence signal from each pixel in the image to a point in the phasor plot. The coordinates $g$ and $s$ (sine and 
cosine transforms, respectively) of each point in the phasor plot are calculated from the fluorescence-intensity decay in the corresponding pixel in the FLIM image. Each lifetime is mapped in a phasor plot and all possible single exponential lifetimes lie on the "universal circle," defined as the semicircle going from point $(0,0)$ to point $(1,0)$, with radius $1 / 2$. Point $(1,0)$ corresponds to $\tau=0$, and point $(0,0)$ to $\tau=\infty$.

In the phasor coordinates, single lifetime components are additive because the phasor follows the vector algebra. This means that the combination of two single exponential decays components will generate phasors within the universal circle and lying on a straight line joining the phasors of the two fixed components. The contribution (referred as fraction in the main text) from one component to the lifetime is proportional to the distance of the phasor from the single components. 


\section{Funding Sources}

V.F. and D.F.G acknowledge VILLUM FONDEN for the Villum Young Investigator Grant “Protein Superstructures as Smart Biomaterials (ProSmart)" 2018-2023 (project number: 19175). The financial supports of the University of Palermo (FFR - PROMETA) is also acknowledged.

\section{Acknowledgements}

Authors acknowledge Bente Vestergaard (University of Copenhagen) for inspiring discussions on the ensemble and structural heterogeneity of amyloid species.

\section{Notes}

The authors declare no conflict of interest.

\section{ASSOCIATED CONTENT}

Supplementary Information. Supporting data on fluorescence microscopy, lifetime imaging analysis and micro-FTIR-

\section{AUTHOR INFORMATION}

\section{Corresponding Author}

*valeria.vetri@unipa.it*vito.fodera@sund.ku.dk

\section{Author Contributions}


VF Conceptualization, Methodology, Validation, Supervision, Data curation, Writing - Original Draft Writing - Review \& Editing. Project administration, Funding acquisition, Resources

VV Conceptualization, Methodology, Validation, Investigation, Formal analysis, Supervision, Writing - Original Draft Writing - Review \& Editing. Project administration, Funding acquisition Resources

GS Investigation, visualization. Review \& Editing,

ML Conceptualization, Methodology, Writing - Review \& Editing, Resources

GDL and DFG Investigation, Formal analysis, Methodology, Review \& Editing, Data curation, visualization.

$\ddagger$ G.D.L and D.F.G. contributed equally to this work. 
References

(1) Ping, Y.; Ding, D.; Ramos, R. A. N. S.; Mohanram, H.; Deepankumar, K.; Gao, J.; Tang, G.; Miserez, A. Supramolecular $\beta$ - Sheets Stabilized Protein Nanocarriers for Drug Delivery and Gene Transfection. ACS Nano 2017. https://doi.org/10.1021/acsnano.6b08393.

(2) Gras, S. L.; Tickler, A. K.; Squires, A. M.; Devlin, G. L.; Horton, M. A.; Dobson, C. M.; MacPhee, C. E. Functionalised Amyloid Fibrils for Roles in Cell Adhesion. Biomaterials 2008, 29 (11), 1553-1562. https://doi.org/10.1016/j.biomaterials.2007.11.028.

(3) Das, S.; Jacob, R. S.; Patel, K.; Singh, N.; Maji, S. K. Amyloid Fibrils: Versatile Biomaterials for Cell Adhesion and Tissue Engineering Applications. Biomacromolecules 2018, 19 (6), 1826-1839. https://doi.org/10.1021/acs.biomac.8b00279.

(4) Shimanovich, U.; Efimov, I.; Mason, T. O.; Flagmeier, P.; Buell, A. K.; Gedanken, A.; Linse, S.; Åkerfeldt, K. S.; Dobson, C. M.; Weitz, D. A.; et al. Protein Microgels from Amyloid Fibril Networks. ACS Nano 2015, 9 (1), 43-51. https://doi.org/10.1021/nn504869d.

(5) Mankar, S.; Anoop, A.; Sen, S.; Maji, S. K. Nanomaterials: Amyloids Reflect Their Brighter Side. Nano Rev. 2011, 2 (1), 6032. https://doi.org/10.3402/nano.v2i0.6032.

(6) Tanaka, H.; Herland, A.; Lindgren, L. J.; Tsutsui, T.; Andersson, M. R.; Inganäs, O. Enhanced Current Efficiency from Bio-Organic Light-Emitting Diodes Using Decorated Amyloid Fibrils with Conjugated Polymer. Nano Lett. 2008, 8 (9), 2858-2861. https://doi.org/10.1021/n1801510z.

(7) Bolisetty, S.; Mezzenga, R. Amyloid-Carbon Hybrid Membranes for Universal Water Purification. Nat. Nanotechnol. 2016, $11 \quad$ (4), 365-371. https://doi.org/10.1038/nnano.2015.310.

(8) Scheibel, T.; Parthasarathy, R.; Sawicki, G.; Lin, X. M.; Jaeger, H.; Lindquist, S. L. Conducting Nanowires Built by Controlled Self-Assembly of Amyloid Fibers and Selective Metal Deposition. Proc. Natl. Acad. Sci. U. S. A. 2003, 100 (8), 4527-4532. https://doi.org/10.1073/pnas.0431081100.

(9) Filipe, V.; Hawe, A.; Schellekens, H.; Jiskoot, W. Aggregation and Immunogenicity of Therapeutic Proteins. Aggreg. Ther. Proteins 2010, 403-433. https://doi.org/10.1002/9780470769829.ch10.

(10) Ow, S. Y.; Dunstan, D. E. A Brief Overview of Amyloids and Alzheimer's Disease. Protein Sci. 2014, 23 (10), 1315-1331. https://doi.org/10.1002/pro.2524.

(11) Dobson, C. M. Protein Folding and Misfolding. Nature 2003, 426. DOI: 10.1038/nature02261

(12) Ross, C. A.; Poirier, M. A. Protein Aggregation and Neurodegenerative Disease. Nat. Med. 
2004, 10 Suppl (July), S10-7. https://doi.org/10.1038/nm1066.

(13) Makin, O. S.; Atkins, E.; Sikorski, P.; Johansson, J.; Serpell, L. C. Molecular Basis for Amyloid Fibril Formation and Stability. Proc. Natl. Acad. Sci. U. S. A. 2005, 102 (2), 315320. https://doi.org/10.1073/pnas.0406847102.

(14) Adamcik, J.; Mezzenga, R. Amyloid Polymorphism in the Protein Folding and Aggregation Energy Landscape. Angew. Chemie - Int. Ed. 2018, 57 (28), 8370-8382. https://doi.org/10.1002/anie.201713416.

(15) Vetri, V.; Leone, M.; Morozova-Roche, L. A.; Vestergaard, B.; Foderà, V. Unlocked Concanavalin A Forms Amyloid-like Fibrils from Coagulation of Long-Lived "Crinkled" Intermediates. PLoS One 2013, 8 (7). https://doi.org/10.1371/journal.pone.0068912.

(16) Carrotta, R.; Vetri, V.; Librizzi, F.; Martorana, V.; Militello, V.; Leone, M. Amyloid Fibrils Formation of Concanavalin A at Basic PH. J. Phys. Chem. B 2011, 115 (12), 2691-2698. https://doi.org/10.1021/jp1042409.

(17) Andersen, C. B.; Hicks, M. R.; Vetri, V.; Vandahl, B.; Rahbek-Nielsen, H.; Thøgersen, H.; Thøgersen, I. B.; Enghild, J. J.; Serpell, L. C.; Rischel, C.; et al. Glucagon Fibril Polymorphism Reflects Differences in Protofilament Backbone Structure. J. Mol. Biol. 2010, 397 (4), 932-946. https://doi.org/10.1016/j.jmb.2010.02.012.

(18) Gelenter, M. D.; Smith, K. J.; Liao, S. Y.; Mandala, V. S.; Dregni, A. J.; Lamm, M. S.; Tian, Y.; Xu, W.; Pochan, D. J.; Tucker, T. J.; et al. The Peptide Hormone Glucagon Forms Amyloid Fibrils with Two Coexisting $\beta$-Strand Conformations. Nat. Struct. Mol. Biol. 2019, 26 (7), 592-598. https://doi.org/10.1038/s41594-019-0238-6.

(19) Heise, H.; Hoyer, W.; Becker, S.; Andronesi, O. C.; Riedel, D.; Baldus, M. Molecular-Level Secondary Structure, Polymorphism, and Dynamics of Full-Length $\alpha$-Synuclein Fibrils Studied by Solid-State NMR. Proc. Natl. Acad. Sci. U. S. A. 2005, 102 (44), 15871-15876. https://doi.org/10.1073/pnas.0506109102.

(20) Salinas, N.; Colletier, J. P.; Moshe, A.; Landau, M. Extreme Amyloid Polymorphism in Staphylococcus Aureus Virulent PSM $\alpha$ Peptides. Nat. Commun. 2018, 9 (1). https://doi.org/10.1038/s41467-018-05490-0.

(21) Madsen, J.; Christiansen, G.; Giehm, L.; Otzen, D. Release of Pharmaceutical Peptides in an Aggregated State: Using Fibrillar Polymorphism to Modulate Release Levels. Colloids and Interfaces 2019, 3 (1), 42. https://doi.org/10.3390/colloids3010042.

(22) Skamris, T.; Marasini, C.; Madsen, K. L.; Foderà, V.; Vestergaard, B. Early Stage AlphaSynuclein Amyloid Fibrils Are Reservoirs of Membrane-Binding Species. Sci. Rep. 2019, 9 (1). https://doi.org/10.1038/s41598-018-38271-2.

(23) Fändrich, M.; Nyström, S.; Nilsson, K. P. R.; Böckmann, A.; LeVine, H.; Hammarström, P. Amyloid Fibril Polymorphism: A Challenge for Molecular Imaging and Therapy. $J$. Intern. Med. 2018, 283 (3), 218-237. https://doi.org/10.1111/joim.12732.

(24) Tycko, R. Amyloid Polymorphism: Structural Basis and Neurobiological Relevance. 
Neuron 2015, 86 (3), 632-645. https://doi.org/10.1016/j.neuron.2015.03.017.

(25) Knowles, T. P. J.; Mezzenga, R. Amyloid Fibrils as Building Blocks for Natural and Artificial Functional Materials. Adv. Mater. 2016, 28 (31), 6546-6561. https://doi.org/10.1002/adma.201505961.

(26) Schack, M. M.; Møller, E. H.; Friderichsen, A. V.; Carpenter, J. F.; Rades, T.; Groenning, M. Optimization of Infrared Microscopy to Assess Secondary Structure of Insulin Molecules Within Individual Subvisible Particles in Aqueous Formulations. J. Pharm. Sci. 2019, 108 (3), 1117-1129. https://doi.org/10.1016/j.xphs.2018.10.028.

(27) Condello, C.; Lemmin, T.; Stöhr, J.; Nick, M.; Wu, Y.; Maxwell, A. M.; Watts, J. C.; Caro, C. D.; Oehler, A.; Keene, C. D.; et al. Structural Heterogeneity and Intersubject Variability of A $\beta$ in Familial and Sporadic Alzheimer's Disease. Proc. Natl. Acad. Sci. U. S. A. 2018 , 115 (4), E782-E791. https://doi.org/10.1073/pnas.1714966115.

(28) Rasmussen, J.; Mahler, J.; Beschorner, N.; Kaeser, S. A.; Häsler, L. M.; Baumann, F.; Nyström, S.; Portelius, E.; Blennow, K.; Lashley, T.; et al. Amyloid Polymorphisms Constitute Distinct Clouds of Conformational Variants in Different Etiological Subtypes of Alzheimer's Disease. Proc. Natl. Acad. Sci. U. S. A. 2017, 114 (49), 13018-13023. https://doi.org/10.1073/pnas.1713215114.

(29) Annamalai, K.; Gührs, K. H.; Koehler, R.; Schmidt, M.; Michel, H.; Loos, C.; Gaffney, P. M.; Sigurdson, C. J.; Hegenbart, U.; Schönland, S.; et al. Polymorphism of Amyloid Fibrils in Vivo. Angew. Chemie - Int. Ed. 2016, 55 (15), 4822-4825. https://doi.org/10.1002/anie.201511524.

(30) Foderà, V.; Vetri, V.; Wind, T. S.; Noppe, W.; Cornett, C.; Donald, A. M.; MorozovaRoche, L. A.; Vestergaard, B. Observation of the Early Structural Changes Leading to the Formation of Protein Superstructures. J. Phys. Chem. Lett. 2014, 5 (18), 3254-3258. https://doi.org/10.1021/jz501614e.

(31) Krebs, M. R. H.; Domike, K. R.; Donald, A. M. Protein Aggregation: More than Just Fibrils. Biochem. Soc. Trans. 2009, 37 (4), 682-686. https://doi.org/10.1042/bst0370682.

(32) Vetri, V.; Foderà, V. The Route to Protein Aggregate Superstructures: Particulates and Amyloid-like Spherulites. FEBS Lett. 2015, $589 \quad$ (19), 2448-2463. https://doi.org/10.1016/j.febslet.2015.07.006.

(33) Krebs, M. R. H.; MacPhee, C. E.; Miller, A. F.; Dunlop, I. E.; Dobson, C. M.; Donald, A. M. The Formation of Spherulites by Amyloid Fibrils of Bovine Insulin. Proc. Natl. Acad. Sci. 2004, 101 (40), 14420-14424. https://doi.org/10.1073/pnas.0405933101.

(34) Christopher, E.; Emily, H.; Joanna F, C.; Mark R, D.; Danielle, C.; Athene M, D. Spherulites of A $\beta 42$ in Vitro and in Alzheimer's Disease Christopher. J. Alzheimers Dis. 2010, 20 (4), 1159-1165. https://doi.org/10.3233/JAD-2010-091630.Spherulites.

(35) Vetri, V.; Piccirilli, F.; Krausser, J.; Buscarino, G.; Łapińska, U.; Vestergaard, B.; Zaccone, A.; Foderà, V. Ethanol Controls the Self-Assembly and Mesoscopic Properties of Human 
Insulin Amyloid Spherulites. J. Phys. Chem. B 2018, 122 (12), 3101-3112. https://doi.org/10.1021/acs.jpcb.8b01779.

(36) Zhang, G.; Verdugo-Escamilla, C.; Choquesillo-Lazarte, D.; García-Ruiz, J. M. Thermal Assisted Self-Organization of Calcium Carbonate. Nat. Commun. 2018, 9 (1), 1-7. https://doi.org/10.1038/s41467-018-07658-0.

(37) Crist, B.; Schultz, J. M. Polymer Spherulites: A Critical Review. Prog. Polym. Sci. 2016, 56, 1-63. https://doi.org/10.1016/j.progpolymsci.2015.11.006.

(38) Smith, M. I.; Foderà, V.; Sharp, J. S.; Roberts, C. J.; Donald, A. M. Factors Affecting the Formation of Insulin Amyloid Spherulites. Colloids Surfaces B Biointerfaces 2012, 89 (1), 216-222. https://doi.org/10.1016/j.colsurfb.2011.09.018.

(39) Foderá, V.; Donald, A. M. Tracking the Heterogeneous Distribution of Amyloid Spherulites and Their Population Balance with Free Fibrils. Eur. Phys. J. E 2010, 33 (4), 273-282. https://doi.org/10.1140/epje/i2010-10665-4.

(40) Fitzpatrick, A. W. P.; Park, S. T.; Zewail, A. H. Exceptional Rigidity and Biomechanics of Amyloid Revealed by 4D Electron Microscopy. Proc. Natl. Acad. Sci. U. S. A. 2013, 110 (27), 10976-10981. https://doi.org/10.1073/pnas.1309690110.

(41) Jiang, Y.; Shi. K.; Xia, D.; Wang, S.; Song, T. Cui F. Protein spherulites for sustained release of interferon: preparation, characterization and in vivo evaluation. J Pharm Sci. 2011,100(5), 1913-1922. doi:10.1002/jps.22403.

(42) Cannon, D.; Eichhorn, S. J.; Donald, A. M. Structure of Spherulites in Insulin, $\beta$ Lactoglobulin, and Amyloid $\beta . \quad A C S$ Omega 2016, 1 (5), 915-922. https://doi.org/10.1021/acsomega.6b00208.

(43) Smith, M. I.; Sharp, J. S.; Roberts, C. J. Giant Amyloid Spherulites Reveal Their True Colours. Soft Matter 2012, 8 (14), 3751-3755. https://doi.org/10.1039/c2sm25147g.

(44) Simone Ruggeri, F.; Habchi, J.; Cerreta, A.; Dietler, G. AFM-Based Single Molecule Techniques: Unraveling the Amyloid Pathogenic Species. Curr. Pharm. Des. 2016, 22 (26), 3950-3970. https://doi.org/10.2174/1381612822666160518141911.

(45) Herling, T. W.; Levin, A.; Saar, K. L.; Dobson, C. M.; Knowles, T. P. J. Microfluidic Approaches for Probing Amyloid Assembly and Behaviour. Lab Chip 2018, 18 (7), 9991016. https://doi.org/10.1039/c7lc01241a.

(46) Horrocks, M. H.; Lee, S. F.; Gandhi, S.; Magdalinou, N. K.; Chen, S. W.; Devine, M. J.; Tosatto, L.; Kjaergaard, M.; Beckwith, J. S.; Zetterberg, H.; et al. Single-Molecule Imaging of Individual Amyloid Protein Aggregates in Human Biofluids. ACS Chem. Neurosci. 2016, 7 (3), 399-406. https://doi.org/10.1021/acschemneuro.5b00324.

(47) Ruggeri, F. S.; Charmet, J.; Kartanas, T.; Peter, Q.; Chia, S.; Habchi, J.; Dobson, C. M.; Vendruscolo, M.; Knowles, T. P. J. Microfluidic Deposition for Resolving Single-Molecule Protein Architecture and Heterogeneity. Nat. Commun. 2018, 9 (1). https://doi.org/10.1038/s41467-018-06345-4. 
(48) Van Maarschalkerweerd, A.; Vetri, V.; Vestergaard, B. Cholesterol Facilitates Interactions between $\alpha$-Synuclein Oligomers and Charge-Neutral Membranes. FEBS Lett. 2015, 589 (19), 2661-2667. https://doi.org/10.1016/j.febslet.2015.08.013.

(49) Rao, E.; Foderà, V.; Leone, M.; Vetri, V. Direct Observation of Alpha-Lactalbumin, Adsorption and Incorporation into Lipid Membrane and Formation of Lipid/Protein Hybrid Structures. Biochim. Biophys. Acta - Gen. Subj. 2019, 1863 (5), 784-794. https://doi.org/10.1016/j.bbagen.2019.02.005.

(50) Thompson, A. J.; Herling, T. W.; Kubánková, M.; Vyšniauskas, A.; Knowles, T. P. J.; Kuimova, M. K. Molecular Rotors Provide Insights into Microscopic Structural Changes during Protein Aggregation. J. Phys. Chem. B 2015, 119 (32), 10170-10179. https://doi.org/10.1021/acs.jpcb.5b05099.

(51) Fricano, A.; Librizzi, F.; Rao, E.; Alfano, C.; Vetri, V. Blue Autofluorescence in Protein Aggregates "Lighted on" by UV Induced Oxidation. Biochim. Biophys. Acta - Proteins Proteomics 2019, 1867 (11), 140258. https://doi.org/10.1016/j.bbapap.2019.07.011.

(52) Santangelo, M. G.; Foderà, V.; Militello, V.; Vetri, V. Back to the Oligomeric State: PHInduced Dissolution of Concanavalin A Amyloid-like Fibrils into Non-Native Oligomers. RSC Adv. 2016, 6 (79), 75082-75091. https://doi.org/10.1039/c6ra16690c.

(53) Vetri, V.; Ossato, G.; Militello, V.; Digman, M. A.; Leone, M.; Gratton, E. Fluctuation Methods to Study Protein Aggregation in Live Cells: Concanavalin a Oligomers Formation. Biophys. J. 2011, 100 (3), 774-783. https://doi.org/10.1016/j.bpj.2010.11.089.

(54) Gupta, Y.; Singla, G.; Singla, R. Insulin-Derived Amyloidosis. Indian J. Endocrinol. Metab. 2015, 19 (1), 174-177. https://doi.org/10.4103/2230-8210.146879.

(55) Foderà, V.; Zaccone, A.; Lattuada, M.; Donald, A. M. Electrostatics Controls the Formation of Amyloid Superstructures in Protein Aggregation. Phys. Rev. Lett. 2013, 111 (10), 1-5. https://doi.org/10.1103/PhysRevLett.111.108105.

(56) Digman, M. A.; Caiolfa, V. R.; Zamai, M.; Gratton, E. The Phasor Approach to Fluorescence Lifetime Imaging Analysis. Biophys. J. 2008, 94 (2), L14-6. https://doi.org/10.1529/biophysj.107.120154.

(57) D’Amico, M.; Schirò, G.; Cupane, A.; D’Alfonso, L.; Leone, M.; Militello, V.; Vetri, V. High Fluorescence of Thioflavin T Confined in Mesoporous Silica Xerogels. Langmuir 2013, 29 (32), 10238-10246. https://doi.org/10.1021/la402406g.

(58) Di Carlo, M. G.; Minicozzi, V.; Foderà, V.; Militello, V.; Vetri, V.; Morante, S.; Leone, M. Thioflavin T Templates Amyloid $\beta(1-40)$ Conformation and Aggregation Pathway. Biophys. Chem. 2015, 206, 1-11. https://doi.org/10.1016/j.bpc.2015.06.006.

(59) Foderá, V.; Pagliara, S.; Otto, O.; Keyser, U. F.; Donald, A. M. Microfluidics Reveals a Flow-Induced Large-Scale Polymorphism of Protein Aggregates. J. Phys. Chem. Lett. 2012, 3 (19), 2803-2807. https://doi.org/10.1021/jz301375d.

(60) Rogers, S. S.; Krebs, M. R. H.; Bromley, E. H. C.; Van Der Linden, E.; Donald, A. M. 
Optical Microscopy of Growing Insulin Amyloid Spherulites on Surfaces in Vitro. Biophys. J. 2006, 90 (3), 1043-1054. https://doi.org/10.1529/biophysj.105.072660.

(61) Piccirilli, F.; Schirò, G.; Vetri, V.; Lupi, S.; Perucchi, A.; Militello, V. Decoding Vibrational States of Concanavalin A Amyloid Fibrils. Biophys. Chem. 2015, 199, 17-24. https://doi.org/10.1016/j.bpc.2015.02.007.

(62) Militello, V.; Casarino, C.; Emanuele, A.; Giostra, A.; Pullara, F. Aggregation Kinetics of Bovine Serum Albumin Studied by FTIR Spectroscopy and Light Scattering. Biophys. Chem. 2004, 107, 175-187. https://doi.org/10.1016/j.bpc.2003.09.004.

(63) Barth, A. Infrared Spectroscopy of Proteins. Biochim. Biophys. Acta - Bioenerg. 2007, 1767 (9), 1073-1101. https://doi.org/10.1016/j.bbabio.2007.06.004.

(64) Singh, B. R. Basic Aspects of the Technique and Applications of Infrared Spectroscopy of Peptides and Proteins. ACS Symposium Series 2009, 2-37. https://doi.org/10.1021/bk-20000750.ch001.

(65) Heremans, K.; Dirix, C.; Meersman, F.; Pfeiffer, H.; Smeller, L. Pressure-Induced Amorphization in Biopolymers. J. Phys. Condens. Matter 2002, 14 (44 SPEC ISS.), 1147711484. https://doi.org/10.1088/0953-8984/14/44/502.

(66) Claborn, K.; Puklin-Faucher, E.; Kurimoto, M.; Kaminsky, W.; Kahr, B. Circular Dichroism Imaging Microscopy: Application to Enantiomorphous Twinning in Biaxial Crystals of 1,8-Dihydroxyanthraquinone. J. Am. Chem. Soc. 2003, 125 (48), 14825-14831. https://doi.org/10.1021/ja035644w.

(67) Foderà, V.; Van De Weert, M.; Vestergaard, B. Large-Scale Polymorphism and AutoCatalytic Effect in Insulin Fibrillogenesis. Soft Matter 2010, 6 (18), 4413-4419. https://doi.org/10.1039/c0sm00169d.

(68) Sulatskaya, A. I.; Maskevich, A. A.; Kuznetsova, I. M.; Uversky, V. N.; Turoverov, K. K. Fluorescence Quantum Yield of Thioflavin T in Rigid Isotropic Solution and Incorporated into the Amyloid Fibrils. PLoS One 2010, 5 (10), 1-7. https://doi.org/10.1371/journal.pone.0015385.

(69) Stsiapura, V. I.; Maskevich, A. A.; Kuzmitsky, V. A.; Uversky, V. N.; Kuznetsova, I. M.; Turoverov, K. K. Thioflavin T as a Molecular Rotor: Fluorescent Properties of Thioflavin T in Solvents with Different Viscosity. J. Phys. Chem. B 2008, 112 (49), 15893-15902. https://doi.org/10.1021/jp805822c.

(70) Robbins, K. J.; Liu, G.; Selmani, V.; Lazo, N. D. Conformational Analysis of Thioflavin T Bound to the Surface of Amyloid Fibrils. Langmuir 2012, 28 (48), 16490-16495. https://doi.org/10.1021/la303677t.

(71) Wolfe, L. S.; Calabrese, M. F.; Nath, A.; Blaho, D. V.; Miranker, A. D.; Xiong, Y. ProteinInduced Photophysical Changes to the Amyloid Indicator Dye Thioflavin T. Proc. Natl. Acad. Sci. U. S. A. 2010, 107 (39), 16863-16868. https://doi.org/10.1073/pnas.1002867107.

(72) Ivancic, V. A.; Ekanayake, O.; Lazo, N. D. Binding Modes of Thioflavin T on the Surface 
of Amyloid Fibrils Studied by NMR. Chemphyschem 2016, 17 (16), 2461-2464. https://doi.org/10.1002/cphc.201600246.

(73) Biancalana, M.; Koide, S. Molecular Mechanism of Thioflavin-T Binding to Amyloid Fibrils. Biochim. Biophys. Acta - Proteins Proteomics 2010, 1804 (7), 1405-1412. https://doi.org/10.1016/j.bbapap.2010.04.001.

(74) Lindberg, D. J.; Wranne, M. S.; Gilbert Gatty, M.; Westerlund, F.; Esbjörner, E. K. SteadyState and Time-Resolved Thioflavin-T Fluorescence Can Report on Morphological Differences in Amyloid Fibrils Formed by A $\beta(1-40)$ and A $\beta(1-42)$. Biochem. Biophys. Res. Commun. 2015, 458 (2), 418-423. https://doi.org/10.1016/j.bbrc.2015.01.132.

(75) Stringari, C.; Cinquin, A.; Cinquin, O.; Digman, M. A.; Donovan, P. J.; Gratton, E. Phasor Approach to Fluorescence Lifetime Microscopy Distinguishes Different Metabolic States of Germ Cells in a Live Tissue. Proc. Natl. Acad. Sci. 2011, 108 (33), 13582-13587. https://doi.org/10.1073/pnas.1108161108.

(76) Sancataldo, G.; Anselmo, S.; Vetri, V. Phasor-FLIM Analysis of Thioflavin T SelfQuenching in Concanavalin Amyloid Fibrils. Microsc. Res. Tech. 2020. https://doi.org/10.1002/jemt.23472.

(77) Sulatskaya, A. I.; Lavysh, A. V.; Maskevich, A. A.; Kuznetsova, I. M.; Turoverov, K. K. Thioflavin T Fluoresces as Excimer in Highly Concentrated Aqueous Solutions and as Monomer Being Incorporated in Amyloid Fibrils. Sci. Rep. 2017, 7 (1), 1-11. https://doi.org/10.1038/s41598-017-02237-7.

(78) Sidhu, A.; Vaneyck, J.; Blum, C.; Segers-nolten, I. Polymorph-Specific Distribution of Binding Sites Determines Thioflavin-T Fluorescence Intensity in $\alpha$ - Synuclein Fibrils. Amyloid 2018, 25 (3), 189-196. https://doi.org/10.1080/13506129.2018.1517736.

(79) Thirumalai, D.; Reddy, G.; Straub, J. E. Role of Water in Protein Aggregation and Amyloid Polymorphism. Acc. Chem. Res. 2012, 45 (1), 83-92. https://doi.org/10.1021/ar2000869.

(80) Bucciarelli, S.; Sayedi, E. S.; Osella, S.; Trzaskowski, B.; Vissing, K. J.; Vestergaard, B.; Foderà, V. Disentangling the Role of Solvent Polarity and Protein Solvation in Folding and Self-Assembly of $\alpha$-Lactalbumin. J. Colloid Interface Sci. 2020, 561 (xxxx), 749-761. https://doi.org/10.1016/j.jcis.2019.11.051.

(81) Singh, S. K. Impact of Product-Related Factors on Immunogenicity of Biotherapeutics. $J$. Pharm. Sci. 2011, 100 (2), 354-387. https://doi.org/https://doi.org/10.1002/jps.22276.

(82) Lindberg, D. J.; Wenger, A.; Sundin, E.; Wesén, E.; Westerlund, F.; Esbjörner, E. K. Binding of Thioflavin-T to Amyloid Fibrils Leads to Fluorescence Self-Quenching and Fibril Compaction. Biochemistry 2017, $56 \quad$ (16), 2170-2174. https://doi.org/10.1021/acs.biochem.7b00035.

(83) Foderà, V.; Librizzi, F.; Groenning, M.; Van De Weert, M.; Leone, M. Secondary Nucleation and Accessible Surface in Insulin Amyloid Fibril Formation. J. Phys. Chem. B 2008, 112 (12), 3853-3858. https://doi.org/10.1021/jp710131u. 
(84) ISS Fluorescence Instumentation and Biomedical. Fluorescence Lifetime Standards. 1-3. 
